\title{
Preparation of a Simple Electrochemical Voltammetry Implementation System
}

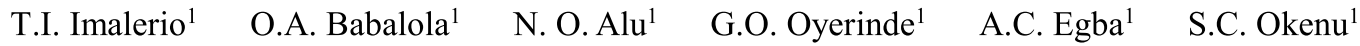 \\ P. O. Oketa ${ }^{2}$ \\ 1.Physics Advanced Research Centre. Sheda Science and Technology Complex, Abuja, Nigeria \\ 2.Dept. of Pure and Applied Physics, Veritas University Abuja Nigeria
}

\begin{abstract}
Cyclic Voltammetry is an Electro-chemical process used in the development Energy Storage cells and general electrochemistry. In this report we present the use of an Atmega 326 microcontroller to prepare a Cyclic Voltammetry (CV) implementation system. The modular design incorporated a software pulse - width modulated triangular wave generator, level shifter circuits, a three - electrode electrochemical tank driver circuit and PLX-DAQ-Excel digital Data Acquisition system. The system measured a $266.1 \Omega$ carbon resistance as $262.7 \Omega$. It also effectively isolated the cathodic redox potential peak $(750 \mathrm{mV})$ and anodic peak $(520 \mathrm{mV})$ of a $1.5 \mathrm{mM}$ Potassium Ferricyanide in $0.1 \mathrm{M}$ Potassium Nitrate at room temperature. A specific capacitance of 11.45 $\mathrm{F} / \mathrm{g}$ was obtained for a $4.4 \mathrm{~cm}^{2}$ coconut shell derived screen printed activated carbon electrode. The design is suitable for basic CV studies in Electrical Energy Storage Devices development.
\end{abstract}

Keywords: Microcontroller, Cyclic Voltammetry, Potentiostat, Level - shifter circuits, Potassium ferricyanide, Energy Storage Devices.

DOI: $10.7176 / \mathrm{JNSR} / 12-18-04$

Publication date:September $30^{\text {th }} 2021$

\section{Introduction}

In the recent past, development of renewable energy resources has attracted global attention. Such energy sources include Solar, Wind, Hydropower, Tidal wave, Ocean thermal and Geothermal. The periodic fluctuations of energy available from these sources make the development of efficient and reliable storage systems imperative. Batteries and related electrochemical devices constitute the core of these storage devices. Research and development of batteries rely substantially on the electrochemical process of voltammetry. It is an electro-analytic process used to identify, quantify and characterize redox active materials (Gopinath \& Russel, 2005). It also finds application in the study of ion adsorption abilities (capacitance) of various electrodes utilized in energy storage devices. When implemented with a standard Potentiostat, the machine imposes stable electrical potential waveforms across a Working Electrode (WE) relative to a Reference Electrode (RE) in an electrochemical cell. It measures the resultant current through a third electrode - the Auxiliary or Counter Electrode (CE). Research efforts in this development have been largely centered on effective stabilization of applied potentials, elimination of electrical noise and accurate measurement of the commonly very low currents. In this regards, Gopinath \& Russel (2005), used Operational Amplifiers (Op. Amp.) and a PIC microcontroller to develop a portable Potentiostat circuit. The design utilized electronic level shifter circuits to interface the analog part of the design to the digital controller. Communication between a personal computer (PC) and the design was through the old RS 232 port. A detailed study of the prevalent potentiostat designs has been reported (Loncaric, 2009). The report involved the development of a system that utilized op. amps. to design the analog sections of the potentiostat. It utilized an Atmega microcontroller and Arduino 0019 compiler to implement USB communication with a PC. In similar format, Aremo et al., (2015) used operational amplifiers in different feedback modules to design a linear sweep voltammetry potentiostat circuit. The data collection was through a PC's DB9 port and it's Virtual Terminal. In broad outlines, literature shows design formats consisting of a combination of analog and digital circuitry with system calibrations using the single electron Ferricyanide ion and Ferro-cyanide ion redox couple. In such analyte studies, the potential of the working electrode is scanned at a specific rate (volts / sec.) to a vertex or switching potential and back to the starting potential in an electrochemical cell. During the process the analyte ions undergo redox activities at the electrode surface. The concentrations of analyte ions at the electrode surface are determined by the Nernst equation

$$
E=E^{\circ \prime}+\frac{0.059}{n} \log \frac{c_{0}}{c_{R}}
$$

1

where $E$ is the applied potential (in V), $E^{0}$ ' is the formal redox potential, $n$ is the number of electrons transferred, and $C s$ is surface concentrations of analyte

The resultant current is plotted against the electrode's potential in a voltammogram. The peak current $i_{p}$ in a voltammogram is given by

$$
i_{p}=\left(2.69 \times 10^{5}\right) n^{3 / 2} A D^{1 / 2} v^{1 / 2} C
$$


where $i_{p}$ is the peak current (in amperes), $n$ is the number of electrons passed per molecule of analyte oxidized or reduced, $\mathrm{A}$ is the electrode area (in $\mathrm{cm}^{2}$ ), D is the diffusion coefficient of analyte (in $\mathrm{cm}^{2} / \mathrm{sec}$ ), $v$ is the potential sweep rate (in volts $/ \mathrm{sec}$ ), and $\mathrm{C}$ is the concentration of analyte in bulk solution (in moles $\left./ \mathrm{cm}^{3}\right)(\mathrm{Bott}$ \& Jackson, 1996).. The separation between the reduction current peak potential $\left(E_{P_{c}}\right)$ and oxidation peak potential $\left(E_{P a}\right)$ in a voltammogram is represented as

$$
\Delta E_{p}=\left|E_{p_{a}}-E_{p_{c}}\right|=\frac{59}{n} m V
$$

where $n$ is the number of electrons per molecule of analyte oxidized or reduced. From eqn.3, a one electron transfer fast reaction thus gives $59 \mathrm{mV}$ separation (Pandarangachar et al., 2010). In evaluation of electrode capacitance by CV, the electrode is immersed in an electrolyte as the WE. Electric double layer form at the electrode / electrolyte interface accessible to the electrolyte ions. Capacitance measurement involves imposing a time varying electric potential on the electrode. The total charge accumulated at the electrode surface is found by integrating the electric current with respect to time. The surface charge density, $\mathrm{q}\left(\mathrm{C} / \mathrm{cm}^{2}\right)$ obtained from the integration of the area under the $\mathrm{I}-\mathrm{V}$ curve is proportional to the capacitance of the electrode since

$$
C\left(V_{f}-V_{i o}\right)=q=\frac{1}{u} \int_{V_{i o}}^{V_{f}} I(V) d V
$$

4

where $\mathrm{C}$ is the total capacitance, $\mathrm{I}$ the current density $\left(\mathrm{A} / \mathrm{cm}^{2}\right)$, $\mathrm{u}$ the sweep rate $(\mathrm{V} / \mathrm{s}), \mathrm{V}_{\mathrm{i} 0}$, the initial and $\mathrm{V}_{\mathrm{f}}$ the final voltages $(\mathrm{V})$. The integral on the right hand side of eqn. (4) is the area under the CV. $\left(V_{f}-V_{i o}\right)$ is the potential window. Thus, the total surface charge, (or total capacitance) of the deposit material can be estimated by evaluating the area under the capacitive current-voltage curve portion of a CV (Kalu et al., 2001).

Unfortunately, the penetration of potentiostats into resource poor laboratories such as prevalent in underdeveloped countries has been very low. In some advanced domains, the development of the instrument has spanned several years. At the present time, they are of varying complexities dictated by their intended applications. One reason for their low penetration in resource poor settings is their costs as even the least expensive commercially available laboratory unit sell for more than one thousand dollars (Rowe et al., 2011). Another observed reason for the low usage is inadequate electronic knowledge required for proper understanding of its generated data. In this paper, we report the development of a Cyclic Voltammetry implementation circuit through the use of cheap and readily available general purpose electronic components. The data generated by the circuit was collected via a PC USB port. The package will serve as a simple and low cost System Development and Training tool for energy storage devices such as Supercapacitors and Batteries.

\section{Materials and Methods}

The circuit was implemented with general purpose electronic components. These were assorted Carbon Resistors, Power step-down transformer $(12 \mathrm{~V}, 500 \mathrm{~mA})$, Voltage regulators (78X... series), Operational Amplifier (uA 741), Two-way Toggle Switch, Electronic Potentiometer, Microcontroller (Atmega 326, Arduino), Dip switch, Capacitors and Breadboard. Other components were Carbon rods, screen printed Activated Carbon electrode, Silver/silver chloride reference electrode and analytical grade (A.R) Potassium Ferricyanide $\left(\left[F_{\theta}(C N)_{6}\right]^{3-}\right)$, Potassium Nitrate and Sulfuric acid. Calibration and Test tools were Digital storage oscilloscope (LW2925B), Digital multimeter (Mastech MY 68), Analog Ammeter (Phywe $1 \mathrm{~mA}-3 \mathrm{~A}, \mathrm{DC} / \mathrm{AC}$ ) and a Personal Computer (PC).

Circuit Design and Calibration. A block layout format of the design is presented in Figure 1.0. The system was powered by $\pm 8.0 \mathrm{VDC}$ obtained from rectified $12 \mathrm{VAC}$ and stabilized with integrated voltage regulators. For simplicity, the power supply section is not represented in the layout. The microcontroller was programmed to provide a variable duty-cycle Pulse Width Modulated (PWM) signal in one of its digital output pins. The PWM signal was converted to a triangular waveform in an R - C low pass filter. The start button and Scan Rate (SR) potentiometer attached to the microcontroller fed input to the code. The generated triangular waveform was level shifted to set reference and peak levels in level shifter A. This module also contained a polarity reversal switch to enable starting potential level switching as is sometimes required in voltammetry studies. Output from this level shifter was fed into the CE and RE driver circuits. The CE supplies the variable tank's current while the RE monitors the tank's constant potentials. This monitored potential is filtered and processed in level shifter $\mathrm{B}$ before feeding to the microcontroller. 


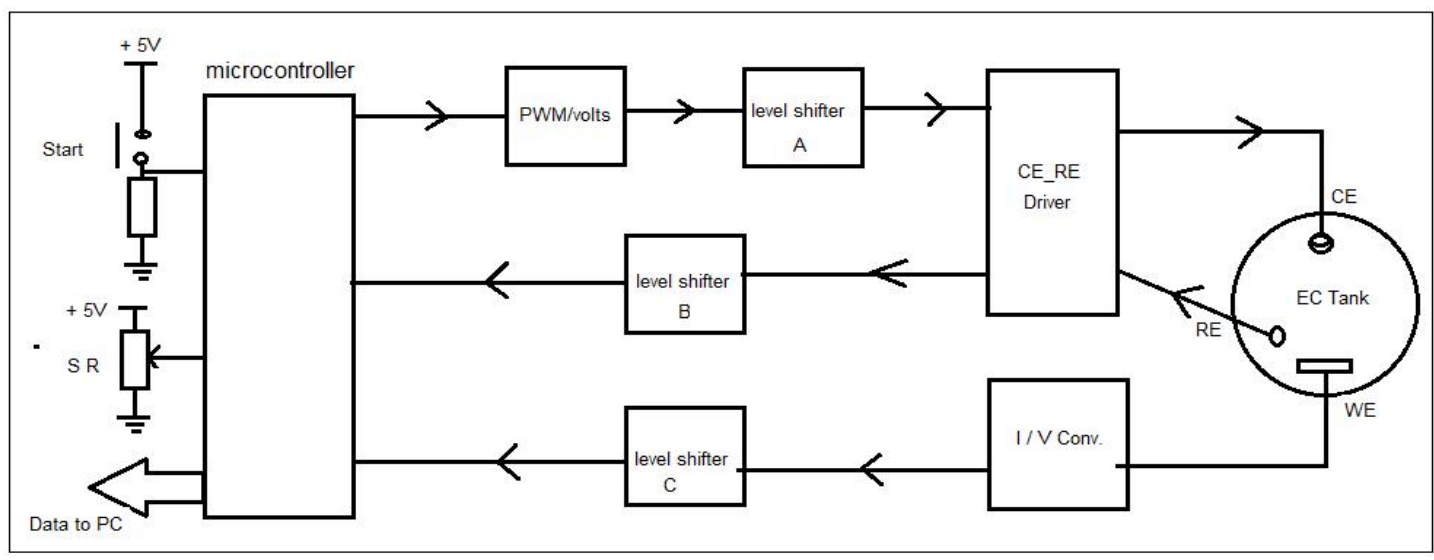

Fig. 1.0 Cyclic Voltammetry implementation system design layout.

The tank's current, conditioned by electroactive activities within the tank and sometimes by redox and ion adsorption activities in WE, is converted to a proportional potential in the Current to Voltage (I/V) converter. This potential is also filtered and conditioned before feeding into the microcontroller. The controller's processed data was finally given to the PC through a USB port.

Pulse - Width - Modulated signal to voltage converter. The Arduino "analogWrite" keyword utilized an external potentiometer input for "Duty - Cycle". This provided the scan rate for the modulated pulses. $\mathrm{R}_{1}$ $(24.1 \mathrm{~K} \Omega)$ and $\mathrm{C}_{1}(0.33 \mathrm{uf})$ were calculated for a chosen cut-off frequency of $20 \mathrm{~Hz}$ for the single pole RC lowpass filter. The chosen $20 \mathrm{~Hz}$ was aimed at the elimination of $50 \mathrm{~Hz}$ mains electricity supply generated noise.

Level - Shifter A and polarity reversal switch. The output from the low- pass filter is unipolar. Voltammetry sometimes require scan voltage polarity reversal. The circuit of Figure 2 includes a voltage range potentiometer $\left(\mathrm{RV}_{1}\right)$, a manual polarity reversal switch and the potential reference setting level shifter $\left(\mathrm{RV}_{2}\right)$. Its output is bipolar. The output voltage $\left(\mathrm{Vo}_{2}\right)$ of $\mathrm{U} 2$ is a function of the inputs at the + and - inputs of the op amp. It is given as (Gopinath \& Russel, 2005).

$$
V_{\text {out }}= \pm\left(\frac{R_{\mathrm{g}}}{R_{\mathrm{z}}}\right)\left(V_{+}-V_{-}\right)
$$

5

It's maximum level is set by $\mathrm{RV}_{1}$. $\mathrm{RV}_{2}$ level shifts the reference to any chosen value between $+5 \mathrm{~V}$ and $-5 \mathrm{~V}$. The values of $50 \mathrm{~K} \Omega$ used for $\mathrm{RV}_{1}$ and $\mathrm{RV}_{2}$ were selected to limit their power dissipations to very low values as only potential levels was needed from them.

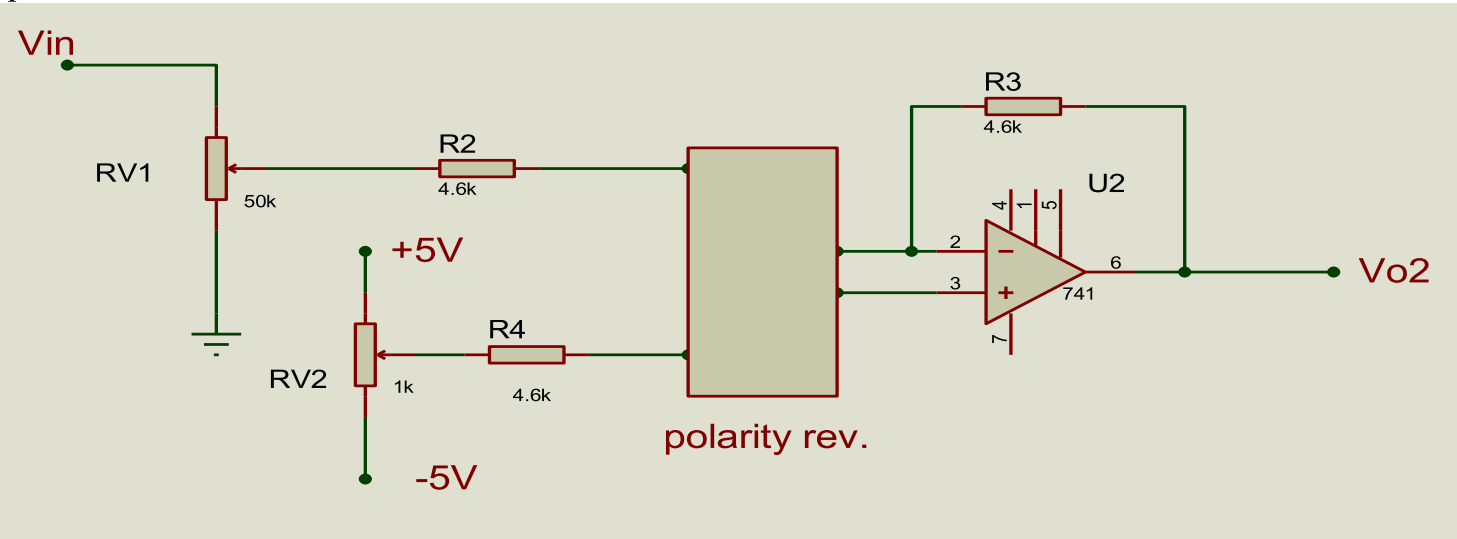

Fig. 2.0 Scan potential range, level - shifter and polarity reversal switch.

For this project, no amplification was needed at this stage. Hence, $\mathrm{R}_{2}=\mathrm{R}_{3}=\mathrm{R}_{4}=4.6 \mathrm{~K} \Omega$. U2 was configured as a unity gain non-inverting or inverting amplifier depending on the position of the polarity reversal switch.

Counter, reference and working electrodes driver circuits. The circuit structure is presented in Figure 3 . It has Reference Electrode amplifier (RE) as U4, Control Electrode amplifier (CE) as U3 and Current to Voltage amplifier (I/E) as U5. The input voltage (Vin) to CE is the output of U2 in Figure 2. The CE and RE are linked through the electrolyte in the electrochemical tank. 


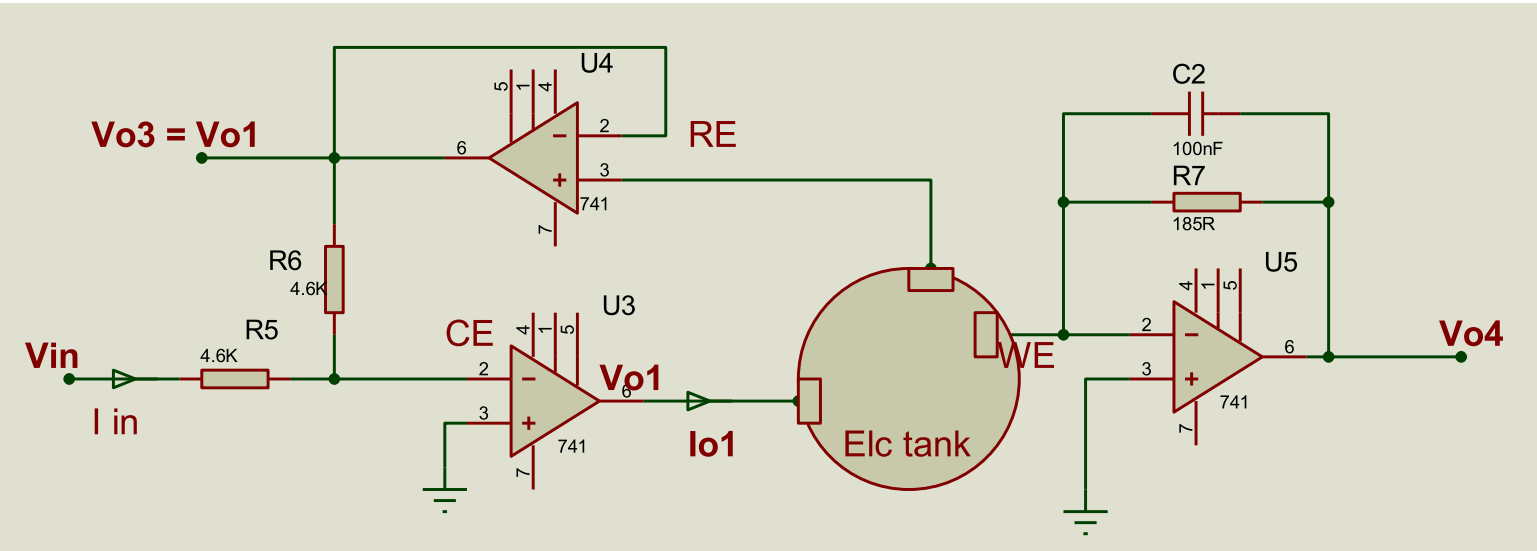

Fig. 3.0 Potentiostat electrodes driver circuits.

The projected maximum output voltage from the potentiostat circuit was 3.0 VDC. Also the expected peak power dissipation of RA was limited to $1.0 \mathrm{~mW}$ as it merely takes the Vol at its high impedance input to its output. Therefore, maximum input current (Iin max) $=0.0003 \mathrm{~A}$, giving $\mathrm{R}_{5}+\mathrm{R}_{6}+75 \Omega=10,000 \Omega$. The nearest preferred resistor values of $4.6 \mathrm{~K} \Omega$ were utilized for $\mathrm{R}_{5}$ and $\mathrm{R}_{6}$.

The current flow in the tank is measured in the electrometer U5 as a voltage drop across the feedback resistor $\mathrm{R}_{7}$. The accuracy of the working electrode current measurement is of prime importance in voltammetry studies. The $\mu \mathrm{A} 741$ operational amplifier utilized in this project was offset nulled by a $10 \mathrm{~K} \Omega$ potentiometer to enhance its signal sensitivity. Assuming zero working electrode impedance, $\mathrm{I}_{\text {tank }}$ and $\mathrm{V}_{04}$ peak values were chosen to be $10 \mathrm{~mA}$ and $2.5 \mathrm{~V}$ respectively. These values gave $250 \Omega$ for $\mathrm{R}_{7}$. In order to provide allowance for the inevitable WE impedance, a lower value of $188 \Omega$ was utilized. The Vo3 and Vo4 were fed into level shifter circuits (not shown) similar to that in Figure 2 before connection to the Analog - to - Digital (ADC) input of the microcontroller.

On construction, the breadboard circuit arrangements was followed by assembly on pre-perforated copper circuit board and then mounted on an earthed metal casing. The earthed metal casing was designed to reduce electromagnetic interference to a minimum.

System calibration and test methods. System calibration was through measurement of a known resistor value, investigation of some known electrochemical properties of Ferricyanide / Ferro-cyanide REDOX couple and capacitance evaluation of a screen printed activated carbon electrode.

The resistance of the carbon resistor was first measured with the digital multi-meter. Figure 4 shows the measurement configuration employed in the test. The potential between CE and RE was scanned from zero to $1.21 \mathrm{~V}$ and back to zero. The upper $1.2 \Omega$ resistor was inserted to provide a slight separation between the current source $\mathrm{CE}$ and the potential monitoring RE.

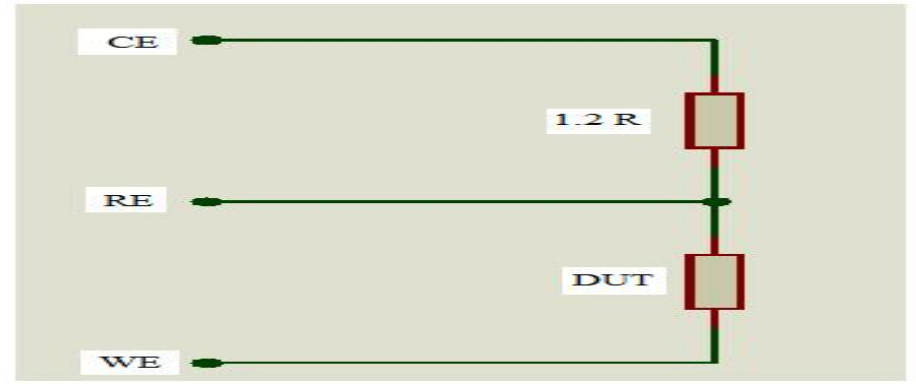

Fig. 4: Potentiostat resistance measurement configuration.

The data of the developed potential across the tested Resistor (Device- Under -Test (DUT)) and its current were collected at the RE and WE amplifiers respectively.

In the ferricyanide investigation, $1.5 \mathrm{mM}$ Potassium Ferricyanide $\left(\left[F_{\theta}(\mathrm{CN})_{6}\right]^{3-}\right)$ in $0.1 \mathrm{M}$ Potassium Nitrate as background electrolyte was utilized (Loncaric c. 2009). The reagents were of analytical grade and solutions prepared with distilled water. Carbon rod served for the counter electrode, while the working electrode was bare carbon prepared in a glassy electrode format $(8.0 \mathrm{~mm}$ diameter). The reference electrode was $A_{g} / A_{g} C l(s) /($ Satd. $K C l)$. A $50 \mathrm{ml}$ beaker covered with a prepared teflon sample / electrodes holder served as the electrochemical cell. The three electrodes were insulated from each other except for the current path though the electrolyte. The excitation signal was varied linearly with time through the scan rate setting of the microcontroller's pwm code. Start / stop potentials were $120 \mathrm{mV}$ and switch point was $0.0 \mathrm{mV}$.

In the activated carbon electrode tests, preparation method of the activated carbon has been discussed 
elsewhere (Imalerio et al., 2014). To form the electrode, the carbon was screen printed on a $4.40 \mathrm{~cm}^{2}$ acid etched aluminum plate with cellulose acetate as binder and dried at $120{ }^{\circ} \mathrm{C}$ for 120 mins. The active electrode mass measured by the digital balance was $0.2940 \mathrm{~g}$. Scan potential window was $0-1.25 \mathrm{VDC}$ at SR of $36 \mathrm{mV} / \mathrm{s}$. Electrolyte was $1.0 \mathrm{M} \mathrm{H}_{2} \mathrm{SO}_{4}$ in distilled water.

\section{Results}

The results below were collected after background corrections of the raw experimental data. The level shifters introduced voltage shifts used to configure the data to the microcontroller compatibility were analytically removed during the corrections.

Figure 5a shows the potential (V) vs time (s) plot of the carbon resistor measurement procedure. The potential was scanned at $37 \mathrm{mV} / \mathrm{s}$ from $0.0 \mathrm{~V}$ to $1.21 \mathrm{~V}$ and back to $0.0 \mathrm{~V}$ in a period of $64.6 \mathrm{~s}$. The resulting current $(\mathrm{mA})$ is plotted vs the potential in Fig. $5 \mathrm{~b}$.
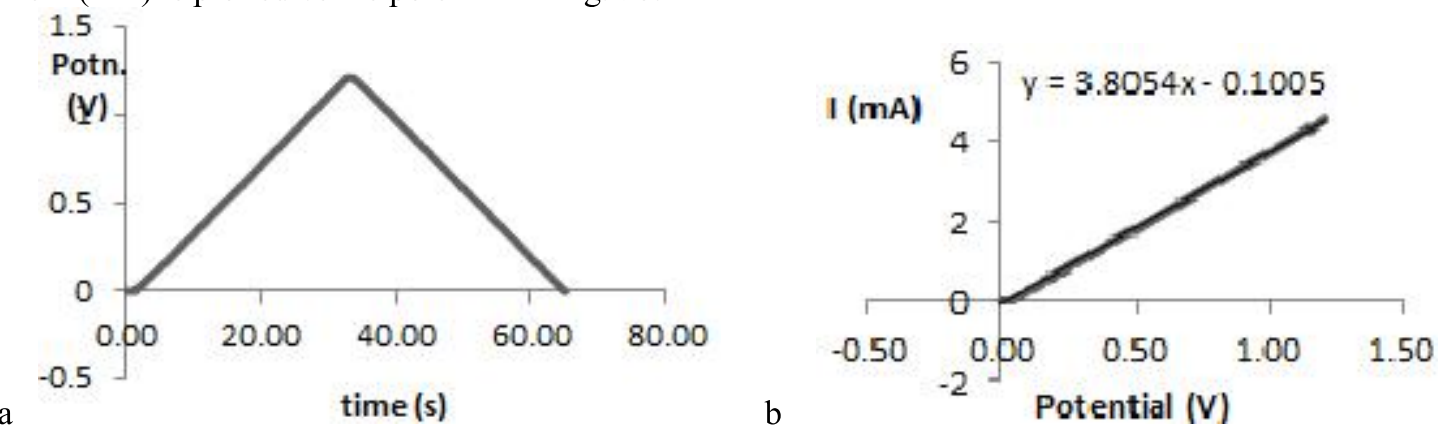

Fig. 5.0 (a) Potential scan for resistance measurement. (b) Plot of current vs potential of carbon resistor. Figure 6 a shows the excitation potential applied to the ferricyanide solution. The potential was scanned at 80 $\mathrm{mV} / \mathrm{s}$ from $1.20 \mathrm{~V}$ to $0.0 \mathrm{~V}$ and back to $1.20 \mathrm{~V}$. The resultant $\mathrm{I} / \mathrm{V}$ plot is shown in Figure $6 \mathrm{~b}$.

a

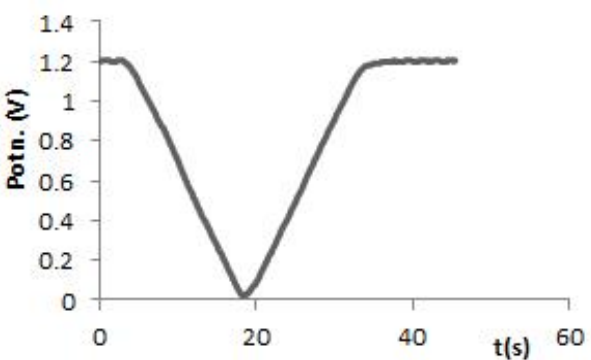

Fig. 6.0 a) Potential scan for CV measurement potassium nitrate background electrolyte.

Figure 7a show the scanned potential. (V) vs time (s) and $7 \mathrm{~b}$ the current density $\left(\mathrm{mA} / \mathrm{cm}^{2}\right)$ vs $(\mathrm{V})$ of the 4.4 $\mathrm{cm}^{2}$ activated carbon electrode in $1.0 \mathrm{M}$ sulfuric acid. The SR was $36 \mathrm{mV} / \mathrm{s}$. The plot's integral area evaluated with Origin 6.1 software was $0.30235 \mathrm{~cm}^{2}$.

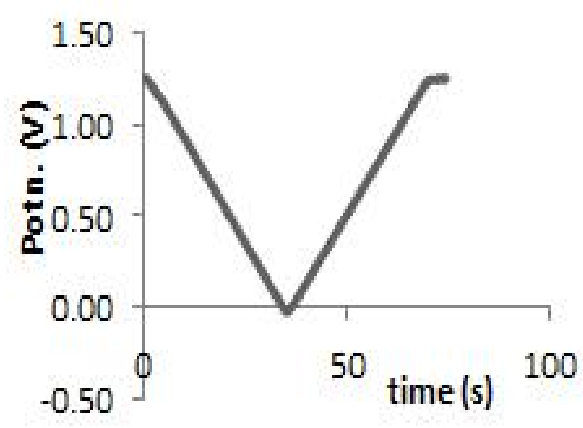

Fig. 7.0 (a, b) CV scan potential and I/V plot of activated carbon electrode in Sulfuric acid electrolyte.

\section{Discussion of Result}

Inset in Figure $5 \mathrm{~b}$ is the Excel software generated linear representative equation of the resistor scan test. With the current in amperes, the measured resistance value is the inverse of the gradient of the plot. It gave $262.7 \Omega$ for the $266.1 \Omega$ resistor. Both the forward and reverse plots are linear and superimposed. It is indicative of zero charge accumulation by the DUT. Resistors do not store charge. 
In Figure $6 \mathrm{~B}$, the reduction process occurred from point $(\mathrm{A})$ the initial potential, to $(\mathrm{C})$ the switching potential. The cathodic current peak $\left(\mathrm{E}_{\mathrm{pc}}\right)$, was at $(\mathrm{B}) 750 \mathrm{mV}$. The oxidation of the generated ferro-cyanide ions back to ferricyanide ions occurred between $(\mathrm{C})$ and $(\mathrm{E})$ with the anodic current peak $\left(\mathrm{E}_{\mathrm{pa}}\right)$ at $(\mathrm{D}) 530 \mathrm{mV}$. The depressions beside the two peaks indicate exhaustion of the respective ions adjacent to the electrode surface. A peak separation $\Delta E_{p}=220 \mathrm{mV}$ was thus recorded from the test

As stated in eqn. 3, $\Delta \mathrm{Ep}$ for a one electron redox couple such as the ferricyanide / ferricyanide couple is theoretically $59 \mathrm{mV}$. A value of $69 \mathrm{mV}$ has been reported for the couple from bare carbon paste glassy carbon electrode through the use of a Model 201 Electroanalyser (EA-201, Chemilink System) in KCL background electrolyte. (Pandarangachar et al., 2010). The substantial deviation $\left(\Delta \mathrm{E}_{\mathrm{p}}=220 \mathrm{mV}\right)$ recorded here was attributed largely to use of ordinary carbon rod in place of a glassy carbon electrode, chemical impurities, difference in background electrolyte and circuit design imperfections.

The increase in current recorded close to the stopping potential (point E) was partially due to the setting in of hydrolysis of water as the electrodes potential was in the region of electrochemical potential window of water.

The drop in current between points $\mathrm{E}$ and $\mathrm{A}$ was the system natural recovery to initial conditions. It was however not possible to return the current to full zero as there was always some residual current flowing despite stirring. Bott \& Jackson (1996) explained this phenomenon to be due to remains of a significant concentration of the active ions close to the electrode surface. This is because not all of the ions that diffused away from the electrode surface during the first part of the scan have been able to diffuse back.

Using the evaluated integral area of $0.30235 \mathrm{~cm}^{2}$ for the screen printed coconut shell derived activated carbon electrode, specific capacitance evaluation through eqn. 4 gave $11.5 \mathrm{~F} / \mathrm{g}$. Fig. $7 \mathrm{~b}$ in contrast to Fig. $5 \mathrm{~b}$ gave clear evidence of charge accumulation at the solid electrode surface. The hysteresis-like shape of Fig. $7 \mathrm{~b}$ is due to electrolyte ions adhesion at the activated carbon surface. However, the enclosed area in Fig. $6 \mathrm{~b}$ was not a consequence of charge accumulation but that of reduction and oxidation processes (pseudo capacitance). Further, the integral area recorded for the screen printed electrode was an indication of some charge accumulation by the electrode as expected.

\section{Conclusion}

In this study, an experimental microcontroller driven voltammetry implementation electronic circuit has been designed and constructed. Basic tests at zero frequency (dc) potential level were successfully carried out on a resistor, a redox active compound (potassium ferricyanide) and a solid activated carbon electrode. The obtained results indicate usefulness of the package as preliminary test equipment for cyclic voltammetry processes in electrochemical studies. Further development will however be needed in fine - tuning and extension of its capabilities to Galvano static - Charge - Discharge (GSD) and Electrochemical Impedance Spectroscopy (EIS).

\section{References}

Aremo B., Adeoye M.O., Obioh I.B., Adeboye O. A. (2015). A simplified microcontroller based potentiostat for low resource applications. Open Journal of Metal (5) : 37 - 46.

Bott A.W., and Brad F. Jackson. (1996). Study of Ferricyanide by Cyclic Voltammetry using the CV-50W. Current Separations 15(1): 25 - 30.

Gopinath, A. V. and Russell, D. (2005). An inexpensive field portable programmable Potentiostat. The Chemical Educator. 11 (1).

Imalerio, T.I., A. N. Amah, D.A. Onoja. (2014). Capacitance and Impedance Evaluations in Coconut Shells and Rice Husks Derived Activated Carbon Electrodes. Journal of Natural Sciences Research. 4 (16): 104 - 108.

Kalu E. E., T.T. Nwoga, V. Srinivasan, J.W. Weidner. (2001). Cyclic voltametric studies of the effects of time and temperature on the capacitance of electrochemically deposited nickel hydroxide. Journal of Power Sources (92): $163-167$.

Loncaric C. (2009). Design, Construction, and Testing of a compact USB powered potentiostat for biosensor applications. Thesis submitted in partial fulfillment of the requirements for the degree of Master of Applied Science in the School of Engineering science, Simon Fraser University Canada.

Pandarangachar M., Kumara S. B. E., Chandraskeckar B. N., Gilbert Ongera, Reddy Satish, Sherigara B. S. (2010). Electrochemical Investigation of Potassium Ferricyanide and Dopamine by 1 - butyl - 4 methylpyridinum tetraflouro borate modified Carbon paste Electrode : A Cyclic Voltammetric study. Int. J. Electrochem. Sci. (5): $1187-1202$

Rowe A. A., Bonham A. J., White R. J., Zimmer M. P., Yadgar R.I., Hobza T. M. (2011). Cheapstat : An open source "Do - it - Yourself" Potentiostat for Analytical and Electrochemical applications. Plus one 6 (9) : e 23783.

STMicroelectronics, uA741 Data Sheet. (2011): http://www. st.com 